\title{
An increased frequency of NK cell receptor and $H L A-C$ group 1 combinations in early-onset type 1 diabetes
}

\author{
K. L. Mehers • A. E. Long • A. R. van der Slik • \\ R. J. Aitken - V. Nathwani - F. S. Wong $\cdot$ S. Bain • \\ G. Gill • B. O. Roep • P. J. Bingley • K. M. Gillespie
}

Received: 25 March 2011 / Accepted: 21 July 2011 /Published online: 10 September 2011

(C) Springer-Verlag 2011

\begin{abstract}
Aims/hypothesis Natural killer (NK) cells serve as primary immune surveillance and are partially regulated by combinations of killer immunoglobulin-like receptor (KIR) genes and their HLA class I ligands. Alterations in NK cell activity have been associated with type 1 diabetes. The aim of this study was to determine whether KIR-HLA class I gene frequency: (1) is altered in a current population with type 1 diabetes compared with healthy controls; and (2) has changed over the half century in which the incidence of type 1 diabetes has increased rapidly.
\end{abstract}

Electronic supplementary material The online version of this article (doi:10.1007/s00125-011-2299-x) contains peer-reviewed but unedited supplementary material, which is available to authorised users.

K. L. Mehers · A. E. Long • R. J. Aitken • V. Nathwani •

P. J. Bingley $\cdot$ K. M. Gillespie $(\square)$

Diabetes and Metabolism, Learning and Research,

University of Bristol, Southmead Hospital,

Bristol, UK

e-mail: K.M.Gillespie@bristol.ac.uk

A. R. van der Slik • B. O. Roep

University of Leiden Medical Centre,

Leiden, the Netherlands

F. S. Wong

Centre for Endocrine and Diabetes Sciences, Cardiff University,

Cardiff, UK

\section{S. Bain}

Institute of Life Sciences, College of Medicine,

Swansea University,

Swansea, UK

G. Gill

Dept of Diabetes/Endocrinology, University of Liverpool,

Liverpool, UK
Methods KIR-HLA class I gene frequencies were compared in 551 individuals diagnosed with type 1 diabetes $\leq 15$ years of age (394 in a current cohort and 157 from the historical 'Golden Years' cohort) and 168 healthy controls. The overall balance of activation and inhibition was analysed using KIR-HLA genotype models.

Results Children with type 1 diabetes who were positive for KIR2DS2/KIR2DL2 and KIR2DL3 were more often homozygous for $H L A-C$ group 1 and this effect was strongest in children diagnosed with diabetes before the age of 5 years $\left(p=0.003\right.$, corrected $\left.p\left[p_{\text {corr }}\right]=0.012\right)$ and $\left(p=0.001, p_{\text {corr }}=\right.$ $0.004)$, respectively. Children with type 1 diabetes have fewer inhibitory KIRs with their corresponding ligands compared with healthy controls $\left(p=1.9 \times 10^{-4}\right)$. This pattern of NK activation has not changed significantly in individuals with type 1 diabetes over the last half century.

Conclusions/interpretation Activating combinations of KIR-HLA genes are more frequent in young children with type 1 diabetes diagnosed in the first 5 years of life, suggesting that NK cell responses may be altered in this group.

Keywords Human leucocyte antigen · Killer immunoglobulin-like receptor - Natural killer cell .

Type 1 diabetes

$\begin{array}{ll}\text { Abbreviations } \\ \text { BDA } & \text { British Diabetic Association } \\ \text { BOX } & \text { Bart's Oxford } \\ \text { HLA-C1 } & H L A-C \text { group 1 } \\ \text { HLA-C2 } & H L A-C \text { group 2 } \\ \text { KIR } & \text { Killer immunoglobulin-like receptor } \\ \text { NK } & \text { Natural killer } \\ p_{\text {corr }} & \text { Corrected } p \text { value }\end{array}$




\section{Introduction}

Natural killer (NK) cells mediate the early killing of virally infected cells and tumours and it is increasingly clear that they also contribute to crosstalk between the innate and adaptive immune responses. Increased NK cell activity has been reported in the periphery of individuals with type 1 diabetes [1-6], but a role for these innate immune cells in the pathogenesis of type 1 diabetes has not, however, been elucidated. NK cells act by either activating or inhibiting cytolysis [7] and their activity is controlled by the balance of inhibitory and activating receptors on the cell surface [8]. One set of human NK cell receptors are the killer immunoglobulin-like receptor (KIR) gene family of membrane glycoproteins on chromosome 19 [9], which consists of 16 genes. Each is either inhibitory or activating in function and is polymorphic both in terms of gene content and allelic variation. The order of the KIR genes along the chromosome has been determined for two distinct haplotypes, termed A and B [10] where the A or 'inhibitory' haplotype is defined by the presence of only one activating gene, KIR2DS4, which is not functional in the majority of individuals, whereas the B or 'activating' haplotype has five functional activating KIRs. Some KIRs interact with specific HLA class I molecules: $H L A-C$ group 1 (HLA-C1) molecules (asparagine at position 80 ) serve as the ligand for two inhibitory KIRs KIR2DL2 and KIR2DL3 and possibly one activating receptor, KIR $2 D S 2$, while the activating KIR2DS1 and inhibitory KIR2DL1 signal through HLA-C group 2 (HLA-C2) molecules (lysine at position 80). KIR3DL1 and KIR3DS1 signal through HLA-Bw4. Functional variation is therefore possible, dependent on the combinations of KIR-HLA class I molecules present [11].

KIRs have been associated with a range of autoimmune conditions, such as psoriatic arthritis [12], rheumatoid arthritis [13], ulcerative colitis [14] and primary sclerosing cholangitis [15]. Results from genetic studies of KIRs in type 1 diabetes have been conflicting. Most have focused on the activating receptor gene KIR2DS2 and the inhibitory receptor gene KIR2DL2, which are in complete linkage disequilibrium and share the HLA-C1 ligand. Some have shown associations [16-18], while others have not [19, 20].

Genome-wide association studies of KIRs in type 1 diabetes are not yet available because this region of chromosome 19 does not have a high-coverage single nucleotide polymorphism map. HLA class I, however, is known to be associated with type 1 diabetes, independent of linkage disequilibrium effects with HLA class II [21]. Genetic interactions between functional KIR genes and their HLA ligands were analysed in well-characterised UK populations of 179 children diagnosed with type 1 diabetes before the age of 5 years, 215 children diagnosed with type 1 diabetes between the ages of 5 and 15 years, and 168 healthy controls. As it is well established that HLA class IImediated genetic susceptibility to type 1 diabetes has decreased over the last half century [22, 23], the data obtained in the first part of this study were then compared with KIR-HLA-C1 frequency in a historical cohort of individuals diagnosed in childhood with type 1 diabetes diagnosed early in the 20th century - the 'Golden Years' cohort-to establish whether determinants of NK cell function have changed over a period in which the incidence of type 1 diabetes has been increasing.

\section{Methods}

Study populations

Current type 1 diabetes cohort Individuals with type 1 diabetes (179 of the individuals diagnosed before the age of 5 years and 215 individuals diagnosed between the ages of 5 years and 15 years) were from either the Bart's Oxford (BOX) study [24] $(n=338)$ or the British Diabetic Association (BDA) cohort [25] $(n=56)$.

The BOX study of childhood diabetes is a populationbased family study that, from 1985 to 2002 , has recruited more than $95 \%$ of the families of children who have developed type 1 diabetes before the age of 21 years in the former Oxford Health Authority Region, UK. The study population is $95 \%$ white and the remainder originate mainly from the Indian subcontinent (data from Office of Population Censuses and Surveys for 1991). All cases of type 1 diabetes were referred by diabetes specialists using the WHO 1985 Diabetes Mellitus Study Group report criteria [26] and had a clinical requirement for insulin treatment from diagnosis. Patients with secondary diabetes, known genetic subtypes including MODY, or clinical type 2 diabetes were not included in the study. In all, 338 samples from BOX were used in the analysis reported here.

A total of 56 white individuals were from the BDA 1972-1981 cohort, a collection of individuals diagnosed with type 1 diabetes under the age of 2 years from across the UK, sourced from the BDA register. Clinical data on these patients were collected from questionnaires sent out to the affected individual, the general practitioner and the consultant. These data included $\mathrm{HbA}_{1 \mathrm{c}}$, creatinine, cholesterol, HDL-cholesterol and LDL-cholesterol. Blood sampling was carried out for glutamate decarboxylase and islet cell antigen 2 antibody testing and DNA collection.

Historical type 1 diabetes ('Golden Years') cohort The Golden Years cohort [27] was recruited from a nationwide register of individuals who had been awarded 50 year Nabarro medals by Diabetes UK. Data were available on 
157 medal winners (52\% men) diagnosed when aged younger than 15 years between 1922 and 1946.

Healthy controls Samples were obtained from 168 healthy volunteers: staff within the University of Bristol $(n=103)$ and individuals participating in the Bristol-based Avon Longitudinal Study of Parents and Children $(n=65)$. [28] Participants were white and did not have type 1 diabetes, nor any other autoimmune diseases or family history of type 1 diabetes.

\section{Ethics approval}

Informed consent was provided by all study participants. Each study population was approved by the research ethics committees in all centres involved in sample collection.

BOX family study Leicestershire, Northamptonshire and Rutland REC 2 (REC No 103/134).

$B D A$ under $2 s$ cohort Genetic factors in the development of insulin-dependent (type 1) diabetes: a study of UK patients diagnosed before age 2 years: MREC/01/6/09. These samples were screened for the presence of mutations underlying neonatal diabetes and were negative.

Golden Years study Ethics approval was obtained by Bain and colleagues as detailed previously [27].

Healthy controls Southmead Research Ethics Committee, Bristol (REC No 09/H0102/11).

\section{KIR genotyping}

The KIR genotyping protocol was originally developed by Martin and colleagues and then adapted by Hiby and colleagues $[12,29]$. The protocol tests for a total of $12 \mathrm{KIR}$ genes (KIR2DL1, KIR2DL2, KIR2DL3, KIR2DS1, KIR2DS2, KIR3DL3, KIR2DS4, KIR2DS3, KIR2DS5, $K I R 2 D L 5, K I R 3 D L 1$ and KIR3DS1) with two different primer sets for each gene. The primer sequences used are detailed in electronic supplementary material (ESM) Table 1. Data were validated through successful participation in the International KIR Exchange Workshop 2007-2010 (www.hla.ucla.edu/cellDNA/Cell/programInfo.htm).

\section{HLA class I and II genotyping}

HLA class I and II genotyping was carried out by PCR using a Dynal RELI SSO system (Invitrogen, Paisley, UK). The type 1 diabetes-associated haplotype $H L A-D R B 1 * 04$ -
$D Q B 1^{*} 0302$ was abbreviated to DR4-DQ8, and $H L A$ $D R B 1 * 03-D Q B 1 * 0201$ was abbreviated to $D R 3-D Q 2$. $H L A-C$ groups: the presence of Asn80 (C1) and Lys80 (C2) was defined using HLA-C genotyping data. HLA-C1 was defined by the presence of $\mathrm{Cw} 1, \mathrm{Cw} 3, \mathrm{Cw} 7, \mathrm{Cw} 8$, Cw12, Cw14 and Cw1601. HLA-C2 was defined by the presence of Cw2, Cw4, Cw5, Cw6, Cw15, Cw1602, Cw17 and $\mathrm{Cw} 18$. HLA-B alleles were divided into the groups Bw4 and Bw6 (as described at http://hla.alleles.org). In some cases the two different alleles could not be distinguished by the results, which were therefore not used.

Data analysis

HLA and KIR genotype frequencies were compared in cases and controls by $\chi^{2}$ analysis. Adjustment for multiple comparisons was made by Bonferroni correction. Composite KIR-HLA genotype effects were analysed using combined KIR-HLA genotype models as described in detail by van der Slik et al. [30]. Briefly, the models were assembled using homologous pairs of inhibitory and activating KIR genes: KIR2DL1 and KIR2DS1 (HLA-C2 [Lys80]), KIR2DL2/3 and KIR2DS2 (HLA-C1 [Asn80]), KIR3DL1 and KIR3DS1 (Bw4). In model one, the presence of each inhibitory KIRHLA ligand pair (KIR2DL1-HLA-C2, KIR2DL2/ KIR2DL3-HLA-C1, KIR3DL1-HLA-Bw4) within an individual was rated -1 . In model two, the presence of each activating KIR-HLA pair (KIR2DS1-HLA-C2, KIR2DS2HLA-C1, KIR3SD1-HLA-Bw4) within an individual was rated +1 . Model three hypothesises that activating KIRs are capable of interacting with other molecules in addition to HLA. The presence of each activating KIR (KIR2DS1, $K I R 2 D S 2$ and KIR3DS1) within an individual was rated +1 . The ratio obtained by combining both the inhibitory and activating genotype models described above reflects the effect of the composite KIR-HLA genotypes. Model four comprised composite inhibitory KIR-HLA/activating KIRHLA genotypes and model five comprised composite inhibitory KIR-HLA/activating KIR genotypes.

\section{Results}

Current type 1 diabetes cohort vs controls

KIR gene frequencies The KIR genotyping results are shown in Table 1. No differences were observed between KIR gene frequencies in children with type 1 diabetes compared with healthy individuals for all KIR genes other than KIR2DL3, for which $97.8 \%$ of those diagnosed with type 1 diabetes under the age of 5 years were positive compared with $90.5 \%$ of controls $(p=0.004$ and corrected $\left.p\left[p_{\text {corr }}\right]=0.048\right)$. No difference in the frequencies of the $\mathrm{A}$ 
Table 1 The frequency of KIR genes in children from the current cohort with type 1 diabetes compared with healthy controls

\begin{tabular}{|c|c|c|c|c|}
\hline Gene & All type 1 diabetes $n=394$ & Onset $0-5$ (years) $n=179$ & Onset $5-15$ (years) $n=215$ & Controls $n=168$ \\
\hline$K I R 2 D L 1$ & $386(97.9)$ & $176(98.3)$ & $210(97.7)$ & $168(100)$ \\
\hline$K I R 2 D L 2$ & $208(52.8)$ & $91(50.8)$ & $117(54.4)$ & $90(53.6)$ \\
\hline$K I R 2 D L 3$ & $371(94.2)$ & $175(97.8)$ & $196(91.2)$ & $152(90.5)$ \\
\hline KIR2DL5 & $213(54.1)$ & $91(50.8)$ & $122(56.7)$ & $95(56.5)$ \\
\hline$K I R 3 D L 1$ & $380(96.4)$ & $174(97.2)$ & $206(95.8)$ & $160(95.2)$ \\
\hline$K I R 3 D L 3$ & $394(100)$ & $179(100)$ & $215(100)$ & $168(100)$ \\
\hline$K I R 2 D S 2$ & $208(52.8)$ & $91(50.8)$ & $117(54.4)$ & $90(53.6)$ \\
\hline KIR2DS1 & $169(42.9)$ & $74(41.3)$ & $95(44.2)$ & $70(41.7)$ \\
\hline$K I R 2 D S 3$ & $120(30.5)$ & $49(27.4)$ & $71(33)$ & $56(33.3)$ \\
\hline$K I R 3 D S 1$ & $177(44.9)$ & $73(40.8)$ & $104(48.4)$ & $74(44)$ \\
\hline KIR2DS4 & $377(95.7)$ & $172(96.1)$ & $205(95.3)$ & $159(94.6)$ \\
\hline KIR2DS5 & $136(34.5)$ & $59(33)$ & $77(35.8)$ & $58(34.5)$ \\
\hline
\end{tabular}

Data are shown as $n(\%)$

and $\mathrm{B}$ haplotypes between individuals with type 1 diabetes and controls was observed (data not shown).

KIR signalling through HLA-C1 molecules Individuals with type 1 diabetes who were positive for the activating receptor $K I R 2 D S 2 /$ inhibitory receptor KIR2DL2 more often had the homozygous genotype HLA-C1C1 (Fig. 1 and Table 2). This effect was strongest in children developing type 1 diabetes under the age of 5 years $\left(p=0.003, p_{\text {corr }}=\right.$ $0.012)$. An age-at-onset effect was observed when the frequency of KIR $2 D S 2 / K I R 2 D L 2 / \mathrm{HLA}-\mathrm{C} 1 \mathrm{C} 1$ was compared between children diagnosed under and over the age of 5 years $(p=0.018)$. HLA-C1C1 was also more frequent in KIR2DL3-positive children with type 1 diabetes $(n=164$, $58.7 \%)\left(p=0.002, p_{\text {corr }}=0.008\right)$ particularly those diagnosed

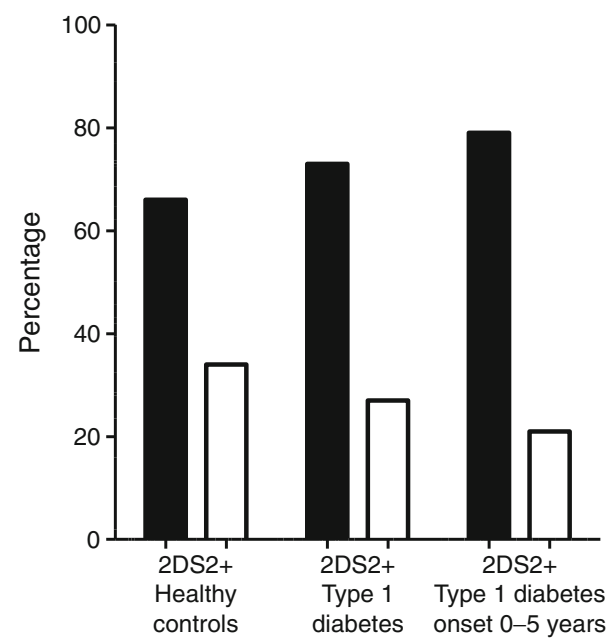

Fig. 1 The frequency of HLA-C1 and HLA-C2 ligands in individuals positive for KIR $2 D L 2 / K I R 2 D S 2$. Healthy controls, $n=85$; all current type 1 diabetes, $n=150$; current type 1 diabetes, onset $0-5$ years, $n=$ 80. Black bars, HLA-C1; white bars, HLA-C2 under the age of $5(n=96,62.3 \%)$ compared with healthy controls $(n=59,42.3 \%)\left(p=0.001, p_{\text {corr }}=0.004\right)$.

At the allelic level the effect was similar: children who developed type 1 diabetes under the age of 5 years and were positive for KIR $2 D S 2 / K I R 2 D L 2$, also had the HLA$\mathrm{C} 1$ allele more often $\left(p=0.006, p_{\text {corr }}=0.02\right)$ compared with healthy controls; a similar result was observed for KIR2DL3.

KIR signalling through HLA-C2 molecules KIR2DL1 (inhibitory) and KIR2DS1 (activating) signal through HLA-C2 but again HLA-C1 was more frequent, indicating decreased capacity for these receptors to signal through their ligands (Table 2).

KIR signalling through HLA-Bw4/6 molecules When the frequency of molecules signalling through HLA-Bw4, KIR3DL1 and KIR3DS1 was analysed, HLA Bw4/Bw4 was less common in both KIR3DL1-positive children with type 1 diabetes and KIR3DS1-positive children with type 1 diabetes, but these data did not reach statistical significance (ESM Table 2).

HLA class II effects As expected the children diagnosed at age under 5 were enriched for the highest-risk genotype $D R B 1 * 04-D Q B 1 * 0302 / D R B 1 * 03-D Q B 1 * 0201 \quad(45.8 \%)$ compared with $29.8 \%$ in the 5-15 years age group and $1.3 \%$ in the control group. Analysis of $K I R-H L A-C$ data by high-risk HLA class II, however, did not significantly strengthen disease associations between KIR and HLA class I (data not shown).

The type 1 diabetes-associated extended haplotype $H L A$ $A 1-B 8-D R 3$ includes HLA-Cw7, which is an HLA-C1 ligand for KIR. To determine whether this extended haplotype had an influence on the data obtained above, 
Table 2 The frequency of KIR molecules signalling through HLA-C1 and HLA-C2 ligands in children with type 1 diabetes compared with healthy controls and the Golden Years cohort

\begin{tabular}{|c|c|c|c|c|c|c|c|}
\hline \multirow[t]{2}{*}{ HLA-C genotype (positive for) } & \multicolumn{3}{|c|}{ Current type 1 diabetes cohort } & \multirow[t]{2}{*}{ Controls } & \multicolumn{3}{|c|}{ Golden Years cohort } \\
\hline & All & Onset $0-5$ years & Onset $5-15$ years & & All & Onset $0-5$ years & Onset $5-15$ years \\
\hline KIR2DL1 (C2 ligand) & $n=289$ & $n=156$ & $n=133$ & $n=156$ & $n=157$ & $n=37$ & $n=120$ \\
\hline $\mathrm{C} 1 \mathrm{C} 1$ & $172(59.5)^{*}$ & $98(62.8)^{*}$ & $74(55.6)$ & $64(41)$ & $80(51)$ & $18(48.6)$ & $62(51.7)$ \\
\hline $\mathrm{C} 1 \mathrm{C} 2$ & $86(29.7)^{*}$ & $42(26.9)$ & $44(33)$ & $74(47.4)$ & $69(43.9)$ & $18(48.6)$ & $51(42.5)$ \\
\hline $\mathrm{C} 2 \mathrm{C} 2$ & $31(10.7)$ & $16(10.2)$ & $15(11.2)$ & $18(11.5)$ & $8(5)$ & $1(2.7)$ & $7(5.8)$ \\
\hline KIR2DL2/ KIRS2 (C1 ligand) & $n=150$ & $n=80$ & $n=70$ & $n=85$ & $n=88$ & $n=20$ & $n=68$ \\
\hline $\mathrm{C} 1 \mathrm{C} 1$ & $86(57.3)$ & $53(66.2)^{\mathrm{a}}$ & $33(47)$ & $37(43.5)$ & $40(45.4)$ & $8(40)$ & $32(47)$ \\
\hline $\mathrm{C} 1 \mathrm{C} 2$ & $48(32)$ & $21(26.2)$ & $27(38.5)$ & $38(44.7)$ & $46(52.3)$ & $12(60)$ & $34(50)$ \\
\hline $\mathrm{C} 2 \mathrm{C} 2$ & $16(10.6)$ & $6(7.5)$ & $10(14.2)$ & $10(11.8)$ & $2(2.2)$ & $0(0)$ & $2(3)$ \\
\hline KIR2DL3 (C1 ligand) & $n=279$ & $n=154$ & $n=125$ & $n=137$ & $n=153$ & $n=37$ & $n=116$ \\
\hline $\mathrm{C} 1 \mathrm{C} 1$ & $164(58.7)^{*}$ & $96(62.3)^{\mathrm{a}}$ & $68(54.4)$ & $58(42.3)$ & $78(51)$ & $18(48.6)$ & $60(51.7)$ \\
\hline $\mathrm{C} 1 \mathrm{C} 2$ & $83(29.7)^{*}$ & $41(26.6)$ & $42(33.6)$ & $63(46)$ & $67(43.8)$ & $18(48.6)$ & $49(42.2)$ \\
\hline $\mathrm{C} 2 \mathrm{C} 2$ & $32(11.4)$ & $17(11)$ & $15(12)$ & $16(11.7)$ & $8(5.2)$ & $1(2.7)$ & $7(6)$ \\
\hline KIR2DS1 (C2 ligand) & $n=127$ & $n=68$ & $n=59$ & $n=67$ & $n=66$ & $n=21$ & $n=45$ \\
\hline $\mathrm{C} 1 \mathrm{C} 1$ & $74(58.2)^{*}$ & $41(60.2)^{*}$ & $33(55.9)$ & $24(35.8)$ & $38(57.6)$ & $12(57.1)$ & $26(57.8)$ \\
\hline $\mathrm{C} 1 \mathrm{C} 2$ & $40(31.5)^{*}$ & $23(33.8)^{*}$ & $17(28.8)^{*}$ & $39(58.2)$ & $28(42.4)$ & $9(42.9)$ & $19(42.2)$ \\
\hline $\mathrm{C} 2 \mathrm{C} 2$ & $13(10.2)$ & $4(5.8)$ & $9(15.2)$ & $4(6)$ & $0(0)$ & $0(0)$ & $0(0)$ \\
\hline
\end{tabular}

Data are shown as $n(\%)$

Significant $\left({ }^{*} p \leq 0.05\right)$ when compared with healthy control frequencies using $\chi^{2}$ analysis and remaining significant following Bonferroni correction

${ }^{a}$ Age-at-onset effect between individuals diagnosed with type 1 diabetes under the age of 5 years and those diagnosed later

the frequency of this haplotype in the type 1 diabetes cohort was examined. Of 398 probands, haplotype data were available for $348(87 \%)$. Of these, $66(19 \%)$ had one copy of the extended DR3-B8-C7 haplotype and nine (3\%) were homozygous. No age-at-onset effects were observed with this haplotype, indicating that the observed association of HLA-C1 with early-onset type 1 diabetes is independent of this extended haplotype.

Overall balance of activation and inhibition Composite KIR-HLA model one [29] demonstrated that individuals with type 1 diabetes have fewer inhibitory KIRs with their corresponding ligands compared with healthy control individuals ( $p=1.9 \times 10^{-4}$; Fig. 2f).

In this model the number of inhibitory KIR2DL1-HLAC2, KIR2DL2/KIR2DL3-HLA-C1 and KIR3DL1-HLABw4 genotypes present within each individual (model one, Fig. 2f) was established. All individuals tested displayed at least one inhibitory KIR-HLA ligand genotype. A single inhibitory KIR-HLA combination, rated -1, was observed more often in patients than controls $(53.9 \%$ vs $31.9 \%$, respectively). All three inhibitory KIR-HLA combinations tested, rated -3 , were less frequently observed in patients than in controls $(15.0 \%$ vs $21.0 \%$, respectively).
The frequencies of the activating homologuesKIR2DS1, KIR2DS2 and KIR3DS1, present in combination with their putative HLA ligands in patients and controlswere compared (model two, Fig. 2g). The number of activating KIR-HLA combinations ranged from none to three combinations in both patients and controls. The combined activating KIR-HLA genotype frequencies in patients did not differ significantly from those in control individuals.

Putative ligands for activating receptors might also include other HLA-like structures or stress-induced cellsurface proteins. The distribution of these activating KIR genes regardless of the presence of HLA between patients and controls were compared (model three, Fig. 2h). Fewer patients were positive for one activating KIR (rated +1 ) compared with controls $(29.7 \%$ vs $36.1 \%$, respectively), whereas individuals who possessed all three activating KIRs were more often patients than controls $(21.0 \%$ vs $15.1 \%$, respectively), but this did not reach statistical significance.

Effector function depends on the combined effect of both inhibitory and activating pathways. Therefore, the inhibition model with either activating KIR-HLA genotypes (model four, Fig. 2i) or activating KIR genotypes (model five, Fig. 2j) were combined. In model four, the composite 
a

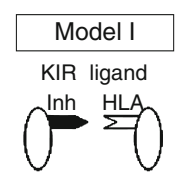

f

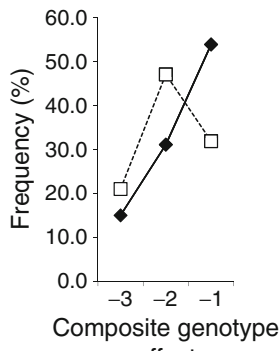

effect

k

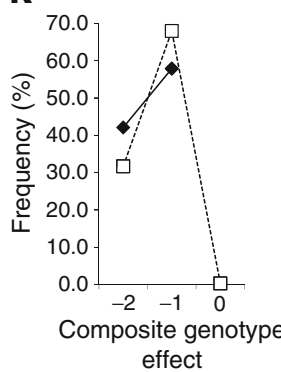

b

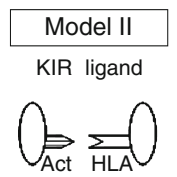

g

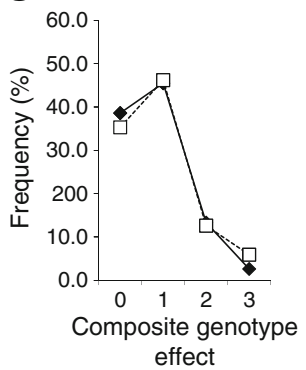

I

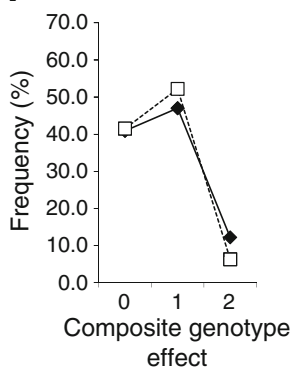

C

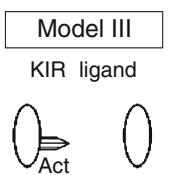

d

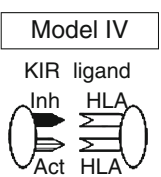

e

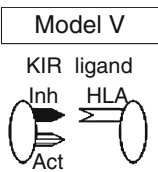

h

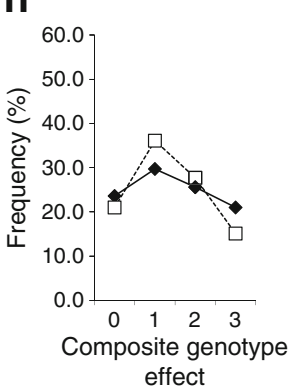

m

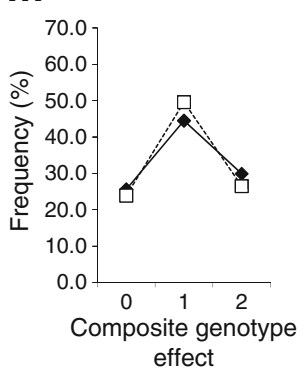

i

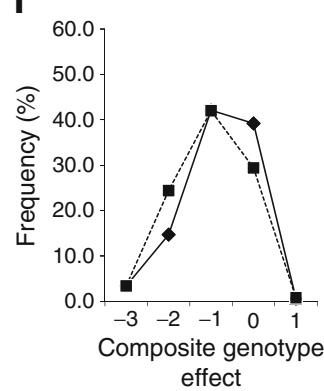

n

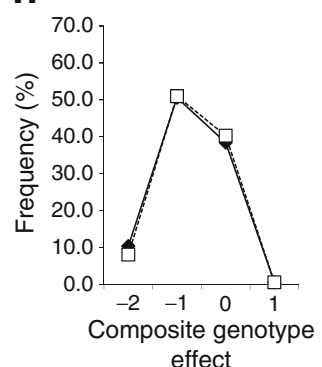

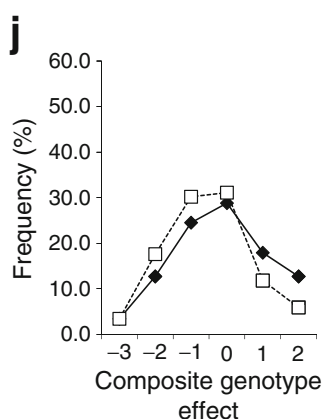

$\mathbf{0}$

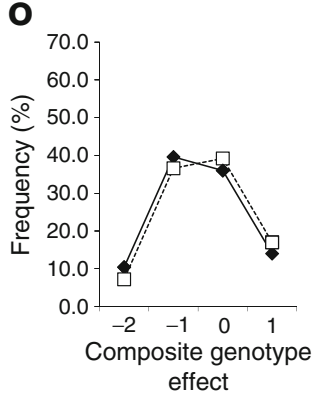

Fig. 2 a-e Schematic representations of models: (a) model one; (b) model two; (c) model three; (d) model four; and (e) model five. $\mathbf{f}-\mathbf{j}$ Comparison of combined KIR-HLA genotype models in current patients ( $n=347$; black diamonds) and controls ( $n=119$; white squares): (f) model one; (g) model two; (h) model three; (i) model four; and (j) model five. k-o Comparison of combined KIR-HLA genotype models in current patients ( $n=347$; white squares) and Golden Years patients ( $n=157$; black diamonds): $(\mathbf{k})$ model one; (l)

inhibitory and activating KIR-HLA genotypes present within individuals ranged from -3 to +1 . Fewer patients had a composite effect of -2 than control individuals $(14.7 \%$ vs 24.4\%), whereas more patients had a composite effect of 0 than controls $(39.2 \%$ vs $29.4 \%)$, though this was not statistically significant. In model five, the inhibitory KIRHLA/activating KIR genotypes present within individuals ranged from -3 to +2 . Fewer patients displayed -2 or -1 than control individuals $(12.7 \%$ vs $17.6 \%$ and $24.5 \%$ vs $30.2 \%$, respectively), whereas more patients displayed +1 or +2 than controls ( $17.9 \%$ vs $11.8 \%$ and $12.7 \%$ vs $5.9 \%$, respectively). There was no difference in frequency for composite genotype +1 and +2 between patients and controls.

Current type 1 diabetes cohort vs historical Golden Years cohort

Overall balance of activation and inhibition The same modelling strategy was applied to data from the current and model two; (m) model three; (n) model four; and (o) model five. Only individuals displaying a complete HLA genotype were included in the analysis. A composite genotype effect of -1 indicates the presence of one inhibitory and +1 indicate the presence of one activating receptor and so on. Composite KIR-ligand genotype frequencies between cases and controls were analysed using a $2 \times n$ test for trend ( $n=$ the number of composite genotypes). For example, model 1, f, $2 \times 3$ : $p=1.9 \times 10^{-4}$. Act, activating; Inh, inhibitory

historical type 1 diabetes cohorts to determine whether there has been a change in the activation pattern over time. The data obtained are also shown in Table 2 and Fig. $2 \mathrm{k}-0$. No significant differences were observed. There was a suggestion that the overall pattern was less activating/more inhibitory in the Golden Years population, but this did not reach statistical significance.

\section{Discussion}

This study shows that children with type 1 diabetes have altered frequencies of functional KIR-HLA class I interactions compared with healthy control individuals. The frequency of HLA-C1, the ligand for three KIR genes (KIR2DL2, KIR2DS2 and KIR2DL3), was increased in children with type 1 diabetes, with the strongest effect in those developing diabetes under the age of 5. It was therefore not surprising that genes signalling through 
HLA-C2 have fewer ligands available in young children with type 1 diabetes.

It is increasingly clear that it is the overall balance of inhibitory or activating KIRs, rather than the effects of individual receptors, that ultimately has the greatest effect on NK cell function. We therefore used multiple composite KIR-HLA models [30] to take into account the cumulative effects of individual KIR-HLA class I and II gene associations in children with type 1 diabetes. These data provided convincing evidence for an overall balance of KIR activation (or lack of inhibition) in young children with type 1 diabetes.

This study represents the largest analysis to date of the entire KIR gene family and their receptors and confirms and expands previous studies showing an increased frequency of KIR2DL2/KIR2DS2/HLA-C1 in type 1 diabetes. The major differences between this study and other studies showing KIR-HLA associations in type 1 diabetes [16-18] is that, consistent with the study of Middleton et al. 2006 [19], we did not see an overall increase in KIR2DS2/ KIR2DL2 in children with type 1 diabetes as reported by van der Slik et al. [16]. More recently Ramos-Lopez et al. (2009) showed, in a combined German/Belgian study of 1,124 patients with type 1 diabetes compared with 716 healthy controls, that a single nucleotide polymorphism (rs2756923) in exon 8 of the inhibitory gene KIR2DL2 was associated with type 1 diabetes [18].

We did, however, observe an association between specific KIR molecules and their $H L A-C$ group ligands. For the first time, we detected a clear age-at-onset effect, with the greatest increase in young children with diabetes. This finding was made possible by using our unique cohort of children diagnosed with type 1 diabetes under the age of 5 years.

Previous analysis, by van der Slik et al., of the KIR gene family and respective HLA class I ligands in 149 children diagnosed with diabetes under the age of 14 in a Dutch population showed an association between KIR2DS2/ KIR2DL2 as well as KIR2DS2/KIR2DL2 and HLA-C1 [16]. Shastry et al. carried out a similar analysis in 98 patients diagnosed with type 1 diabetes under the age of 18 years compared with 70 healthy controls in a Latvian population, showing an association between KIR2DS1/ KIR2DL1, HLA-C1 and type 1 diabetes [17]. In contrast, Santin et al. failed to observe an association between KIR and HLA-C1 in a Basque population with type 1 diabetes [20].

Most studies of KIR genes to date have focused on KIR2DS2/KIR2DL2, yet it is increasingly clear that activating KIRs bind their putative HLA class I ligands with low (e.g. KIR2DS1) to very low (e.g. KIR2DS2) affinity. Recent data [31] suggest that humans are unique among hominid species in appearing not to have a functioning HLA-C1- specific activating receptor. If true, this would indicate that KIR2DL2 and KIR2DL3 signal through HLA-C1 while KIR2DS2 does not. However, as reported by Moesta et al. [31], weak reactivity with $H L A-C^{*} 1601$ showed that KIR2DS2 has narrowed specificity for HLA-C, raising the possibility that it is also highly dependent on the peptide bound by HLA-C. Moesta et al. were therefore unable to rule out the possibility that KIR2DS2 functions in the presence of peptides presented by $H L A-C$. In addition Stewart et al. [32] suggest that some diseases may be associated with only certain combinations of KIR-HLA genes because the action of the activating KIR might be tissue specific due to specific peptide preferences. We therefore included KIR2DS2 in our analysis.

A weakness of the multiple composite KIR-HLA models we used [30] to take into account the cumulative effects of individual KIR-HLA class I gene associations is that they cannot measure the strength of each signal, only the presence of gene-ligand combinations, but this nevertheless provide additional data compared with analysing individual KIR-HLA class I genes in isolation.

We, and others, have already established that the frequency of HLA class II risk genotypes is decreasing as the incidence of type 1 diabetes increases $[22,23]$. We therefore examined whether there has been a concomitant change in the overall balance of genetic determinants of NK cell activation. Our data suggest that there has not been a major change in this balance in children with type 1 diabetes over the last half century. This part of the study was limited by the number of samples available for analysis in the Golden Years cohort, which nevertheless remains one of the rare resources in which to carry out this type of analysis. Analysis of the KIR-HLA data obtained in both the Golden Years and current cohorts showed that the greatest increase in activating combinations is occurring in the population undergoing the most rapid current increase in frequency-children under the age of 5 years in whom the incidence of diabetes is predicted to double between 2005 and 2020 [33].

To date, there have been few functional studies of NK cells in type 1 diabetes in humans. Rodacki and colleagues [34] studied the frequency and activation state of blood NK cells at various stages in human type 1 diabetes and showed that, at diagnosis, NK cells are decreased in absolute number but are unusually activated (as determined by IFN$\gamma$ production). This is consistent with our genetic data showing an increased balance of activating receptors in young children with type 1 diabetes. Wilcox and colleagues demonstrated an increased frequency of enteroviral capsid protein (vp1) in pancreas from an individual with type 1 diabetes, but NK cells were rare [35]. Dotta and colleagues [36] identified enterovirus in three of six pancreases from recent-onset type 1 diabetes cases. Infected islets were 
dominated by NK cell infiltration but beta cell function appeared to be preserved. A recent study by Gur and colleagues identified beta cell ligands for the activating NK cell receptor Nkp46 in human and murine pancreas that were shown to be responsible for diabetes development in the NOD mouse [37]. More functional studies of NK cells in early-onset type 1 diabetes are required to determine the effects of the genetic associations described here.

Acknowledgements The BOX study has been funded by the Wellcome Trust and Diabetes UK. The BDA Under $2 \mathrm{~s}$ genetic collection is funded by Diabetes UK. The Golden Years cohort was funded by the Warren Fund from Diabetes UK. The genotyping carried out in this study was funded by a University of Bristol PhD scholarship to K.L. Mehers.

Contribution statement All the authors (KLM, AEL, ARS, RJA, VN, FSW, SB, GG, BDR, PJB and KMG) contributed to: the conception and design, or analysis and interpretation of data; drafting the article or revising it critically for important intellectual content; and approval of the final version to be published.

Duality of interest The authors declare that there is no duality of interest associated with this manuscript.

\section{References}

1. Herold KC, Huen A, Gould L, Traisman H, Rubenstein AH (1984) Alterations in lymphocyte subpopulations in type 1 (insulin-dependent) diabetes mellitus: exploration of possible mechanisms and relationships to autoimmune phenomena. Diabetologia 27(Suppl):102-105

2. Nair MP, Lewis EW, Schwartz SA (1986) Immunoregulatory dysfunctions in type I diabetes: natural and antibody-dependent cellular cytotoxic activities. J Clin Immunol 6:363-372

3. Negishi K, Waldeck N, Chandy G et al (1986) Natural killer cell and islet killer cell activities in type 1 (insulin-dependent) diabetes. Diabetologia 29:352-357

4. Wilson RG, Anderson J, Shenton BK, White MD, Taylor RM, Proud G (1986) Natural killer cells in insulin dependent diabetes mellitus. Br Med J (Clin Res Ed) 293:244

5. Hussain MJ, Alviggi L, Millward BA, Leslie RD, Pyke DA, Vergani D (1987) Evidence that the reduced number of natural killer cells in type 1 (insulin-dependent) diabetes may be genetically determined. Diabetologia 30:907-911

6. Lorini R, Moretta A, Valtorta A et al (1994) Cytotoxic activity in children with insulin-dependent diabetes mellitus. Diabetes Res Clin Pract 23:37-42

7. Ljunggren HG, Karre K (1990) In search of the 'missing self': MHC molecules and NK cell recognition. Immunol Today $11: 237-244$

8. Lanier LL (1998) NK cell receptors. Annu Rev Immunol 16:359393

9. Carrington M, Norman P (2003) The KIR gene cluster. NCBI. Available at www.ncbi.nlm.nih.gov/books/NBK10135/

10. Uhrberg M, Valiante NM, Shum BP et al (1997) Human diversity in killer cell inhibitory receptor genes. Immunity 7:753-763

11. Vilches C, Parham P (2002) KIR: diverse, rapidly evolving receptors of innate and adaptive immunity. Annu Rev Immunol $20: 217-251$
12. Martin MP, Nelson G, Lee JH et al (2002) Cutting edge: susceptibility to psoriatic arthritis: influence of activating killer Ig-like receptor genes in the absence of specific HLA-C alleles. J Immunol 169:2818-2822

13. Yen JH, Lin CH, Tsai WC et al (2006) Killer cell immunoglobulinlike receptor gene's repertoire in rheumatoid arthritis. Scand J Rheumatol 35:124-127

14. Jones DC, Edgar RS, Ahmad T et al (2006) Killer Ig-like receptor (KIR) genotype and HLA ligand combinations in ulcerative colitis susceptibility. Genes Immun 7:576-582

15. Hov JR, Lleo A, Selmi C et al (2010) Genetic associations in Italian primary sclerosing cholangitis: heterogeneity across Europe defines a critical role for HLA-C. J Hepatol 52:712-717

16. van der Slik AR, Koeleman BP, Verduijn W, Bruining GJ, Roep BO, Giphart MJ (2003) KIR in type 1 diabetes: disparate distribution of activating and inhibitory natural killer cell receptors in patients versus HLA-matched control subjects. Diabetes 52:2639-2642

17. Shastry A, Sedimbi SK, Rajalingam R et al (2008) Combination of KIR 2DL2 and HLA-C1 (Asn) confers susceptibility to type 1 diabetes in Latvians. Int J Immunogenet 35:439-446

18. Ramos-Lopez E, Scholten F, Aminkeng F et al (2009) Association of KIR2DL2 polymorphism rs2756923 with type 1 diabetes and preliminary evidence for lack of inhibition through HLA-C1 ligand binding. Tissue Antigens 73:599-603

19. Middleton D, Halfpenny I, Meenagh A, Williams F, Sivula J, Tuomilehto-Wolf E (2006) Investigation of KIR gene frequencies in type 1 diabetes mellitus. Hum Immunol 67:986-990

20. Santin I, de Nanclares GP, Calvo B, Gaafar A, Castano L, Bilbao JR (2006) Killer cell immunoglobulin-like receptor (KIR) genes in the Basque population: association study of KIR gene contents with type 1 diabetes mellitus. Hum Immunol 67:118-124

21. Nejentsev S, Howson JM, Walker NM et al (2007) Localization of type 1 diabetes susceptibility to the MHC class I genes HLA-B and HLA-A. Nature 450:887-892

22. Gillespie KM, Bain SC, Barnett AH et al (2004) The rising incidence of childhood type 1 diabetes and reduced contribution of high-risk HLA haplotypes. Lancet 364:1699-1700

23. Hermann R, Knip M, Veijola R et al (2003) Temporal changes in the frequencies of HLA genotypes in patients with type 1 diabetes - indication of an increased environmental pressure? Diabetologia 46:420-425

24. Bingley PJ, Gale EA (1989) Incidence of insulin dependent diabetes in England: a study in the Oxford region, 1985-6. BMJ 298:558-560

25. Botha JL, Parker H, Raymond NT, Swift PG (1992) Diabetes diagnosed before the age of 2 years: mortality in a British cohort 8-17 years after onset. Int J Epidemiol 21:1132-1137

26. World Health Organization (1985) Diabetes mellitus: report of a World Health Organization Study Group. World Health Organization, Geneva

27. Bain SC, Gill GV, Dyer PH et al (2003) Characteristics of type 1 diabetes of over 50 years duration (the Golden Years Cohort). Diabet Med 20:808-811

28. Golding J, Pembrey M, Jones R (2001) ALSPAC - the Avon Longitudinal Study of Parents and Children. I Study methodology Paediatr Perinat Epidemiol 15:74-87

29. Hiby SE, Walker JJ, O'Shaughnessy KM et al (2004) Combinations of maternal KIR and fetal HLA-C genes influence the risk of preeclampsia and reproductive success. J Exp Med 200:957-965

30. van der Slik AR, Alizadeh BZ, Koeleman BP, Roep BO, Giphart MJ (2007) Modelling KIR-HLA genotype disparities in type 1 diabetes. Tissue Antigens 69(Suppl 1):101-105

31. Moesta AK, Graef T, Abi-Rached L, Older Aguilar AM, Guethlein LA, Parham P (2010) Humans differ from other 
hominids in lacking an activating $\mathrm{NK}$ cell receptor that recognizes the C1 epitope of MHC class I. J Immunol 185:42334237

32. Stewart CA, Laugier-Anfossi F, Vély F et al (2005) Recognition of peptide-MHC class I complexes by activating killer immunoglobulinlike receptors. Proc Natl Acad Sci USA 102:13224-13229

33. Patterson CC, Dahlquist GG, Gyurus E, Green A, Soltesz G (2009) Incidence trends for childhood type 1 diabetes in Europe during 1989-2003 and predicted new cases 2005-20: a multicentre prospective registration study. Lancet 373:20272033
34. Rodacki M, Svoren B, Butty V et al (2007) Altered natural killer cells in type 1 diabetic patients. Diabetes 56:177-185

35. Willcox A, Richardson SJ, Bone AJ, Foulis AK, Morgan NG (2009) Analysis of islet inflammation in human type 1 diabetes. Clin Exp Immunol 155:173-181

36. Dotta F, Censini S, van Halteren AG et al (2007) Coxsackie B4 virus infection of beta cells and natural killer cell insulitis in recent-onset type 1 diabetic patients. Proc Natl Acad Sci USA 104:5115-5120

37. Gur C, Porgador A, Elboim $\mathrm{M}$ et al (2010) The activating receptor NKp46 is essential for the development of type 1 diabetes. Nat Immunol 11:121-128 